\title{
Activation of Dopamine Cell Firing by Repeated L-DOPA Administration to Dopamine-Depleted Rats: Its Potential Role in Mediating the Therapeutic Response to L-DOPA Treatment
}

\author{
David G. Harden and Anthony A. Grace \\ Departments of Neuroscience and Psychiatry, Center for Neuroscience, University of Pittsburgh, Pittsburgh, \\ Pennsylvania 15260
}

The administration of L-dihydroxyphenylalanine (L-DOPA) to patients with Parkinson's disease is known to produce acute effects that include the reduction of rigidity as well as delayed therapeutic actions involving the resumption of complex motor behavior. In order to examine the potential role of dopamine (DA) cell activity in mediating these responses, the effects of acute and repeated L-DOPA administration on the electrophysiological activity of the residual dopamine (DA) neurons were examined in rats that had received partial 6-hydroxydopamine (6-OHDA)-induced DA lesions. DA cell activity was assessed along three dimensions: (1) the relative proportion of DA neurons exhibiting spontaneous spike firing, (2) their basal firing rate, and (3) their firing pattern. Following 6-OHDA-induced DA depletion, rats were treated for 1 month with saline or L-DOPA. In addition, rats from each group received either an acute injection of L-DOPA or saline on the day of recording. In rats receiving repeated saline treatment, the DA neurons recorded following acute L-DOPA administration were firing at significantly slower basal firing rates and exhibited less burst firing when compared to saline-pretreated rats given acute saline. In contrast, DA cells recorded from rats that had received repeated L-DOPA administration for 4 weeks followed by an acute saline injection did not exhibit any significant differences from DA cells of intact control rats with respect to basal firing rate or firing pattern; however, there was a substantial increase in the proportion of DA neurons exhibiting spontaneous spike firing after correcting for 6-OHDA-induced cell loss. In addition, in rats receiving repeated L-DOPA treatment, the DA cells recorded following acute administration of L-DOPA showed significantly less of a reduction in firing rate when compared to the cells recorded following acute L-DOPA in the saline treatment group. These results show that: (1) acute L-DOPA administration appears to exert its actions by DA autoreceptor stimulation, whereas (2) repeated L-DOPA administration increases the proportion of spontaneously active DA neurons in partially lesioned rats. As a result, repeated

Received Feb. 15, 1995; revised Apr. 26, 1995; accepted May 5, 1995.

We thank Mr. Mark Antkowiak and Dr. Shao-Pii Onn for technical assistance and Hoffmann-LaRuclie Inc. for their generous gift of benserazide $\mathrm{HCl}$. This work was supported by USPHS MH42217, MH45156, NS19608, and MHOI055.

Correspondence should be addressed to David Harden, Department of Neuroscience, 446 Crawford Hall, University of Pittsburgh, Pittsburgh, PA 15260. Copyright 1995 Society for Neuroscience $0270-6474 / 95 / 156157-10 \$ 05.00 / 0$
L-DOPA administration would be expected to cause an increase in spike-dependent DA release as a consequence of the greater proportion of DA cells showing spontaneous activity. This may be the major factor underlying the delayed therapeutic benefits of L-DOPA therapy in the treatment of Parkinson's disease.

[Key words: extracellular recording, in vivo, substantia nigra, 6-hydroxydopamine, L-DOPA, burst firing, firing rate, electrophysiology]

The nigrostriatal dopaminergic projection is known to play a critical role in locomotor function, and degeneration of the dopaminergic system as occurs in Parkinson's disease (Hornykiewicz, 1966) results in a severe disruption of motor behavior. Nonetheless, rapid advances in our understanding of this neurological disorder have been facilitated by the development of an animal model in which intracerebral infusion of the catecholamine neurotoxin 6-hydroxydopamine (6-OHDA) causes a Parkinson-like condition in rats (Zigmond and Stricker, 1984). One of the most remarkable findings revealed by these studies is the extraordinary capacity of the DA system to compensate for extensive damage (Zigmond et al., 1989). Thus, rats that have lost $90 \%$ or more of striatal DA levels will exhibit recovery of behavioral function within 1-2 months following the lesion (Marshall and Teitelbaum, 1973; Zigmond and Stricker, 1973). In a similar manner, patients with Parkinson's disease do not show profound motor impairment until the loss of brain DA levels exceeds 80\% (Bernheimer et al., 1973; Lloyd et al., 1975). Several studies have reported that compensatory changes in the neurochemistry of this system are likely to contribute to the behavioral recovery in rats, including increases in DA synthesis within the remaining DA terminals (Acheson et al., 1980; Zigmond and Stricker, 1984), increased DA release (Agid et al., 1973; Hefti et al., 1980; Stachowiak et al., 1987; Zhang et al., 1988), increased sensitivity of striatal neurons to DA (Creese et al., 1977; Creese and Synder, 1979), and a decrease in the removal of DA from the synapse due to the lesion-induced loss of DA uptake sites on DA terminals (Zigmond et al., 1984).

In addition to the compensatory processes occurring at the site of DA release within the striatum, physiological alterations have also been observed within the cells of origin of the nigrostriatal DA pathway. Previous studies showed that the lesioninduced loss of DA cell bodies is accompanied by a corresponding decrease in the number of spontaneously active DA neurons recorded in the substantia nigra (SN) (Hollerman and Grace, 1990). However, when compared to the percent of DA cells 
counted histologically that remained in rats that had moderate to severe loss of striatal DA levels, the relative proportion of DA neurons exhibiting spontaneous activity as well as their basal firing rate and firing pattern were not altered from control values (Hollerman and Grace, 1990). Alterations in DA cell activity were only observed in rats when the extent of striatal tissue DA depletion was greater than $95 \%$, which also corresponded to the lesion size from which rats typically fail to exhibit behavioral recovery (Zigmond and Stricker, 1984). Although one might predict that lesion-induced increases in striatal activity (Nisenbaum et al., 1986, 1988; Orr et al., 1986) would cause a feedback activation of DA cell firing (Grace and Bunney, 1985), one factor that may have provided an offsetting influence to this striatonigral feedback is proposed to be the substantial increase in the sensitivity of the inhibitory DA cell autoreceptors (Pucak and Grace, 1991). The involvement of somatodendritic autoreceptors in maintaining stability in this system is supported by the finding that administration of what would normally be subthreshold doses of the DA antagonist haloperidol to rats that had recovered from DA lesions leads to profound behavioral akinesia and the induction of depolarization block in the remaining DA cells (Hollerman and Grace, 1989; Hollerman et al., 1992).

The most effective treatment for Parkinson's disease is the administration of the DA precursor L-DOPA. This drug is typically thought to act by pharmacologically restoring DA levels in the striatum (Lloyd et al., 1975). However, both clinical observations as well as in animal studies utilizing DA depletion as a model for Parkinson's disease suggest that the mechanism of action of this drug may be more complex than this model implies. Thus, acute administration of L-DOPA is associated with a robust increase in extracellular DA levels within in the striatum in DA-depleted animals (Spencer and Wooten, 1984; Orosz and Bennet, 1992) which appears to correlate with the initial reduction in rigidity observed in parkinsonian patients (Gancher, 1992; Juncos, 1992). On the other hand, repeated administration of L-DOPA is necessary for the resumption of complex motor behavior (Hardie, 1990; Gancher, 1992; Juncos, 1992). Studies using in vivo microdialysis in rats with unilateral DA depletions have demonstrated that elevated extracellular DA levels in the DA-depleted striatum that occur in response to L-DOPA administration are unaltered during the course of repeated drug administration, even though the behavioral response to L-DOPA is enhanced over a $28 \mathrm{~d}$ period with the administration of successive doses of this drug (Wachtel and Abercrombie, 1993). Therefore, the time course at which L-DOPA administration induces maximal increases in extracellular DA levels in the striatum in rats does not appear to be correlated with its behavioral actions. Other evidence suggests that the site of action of L-DOPA may actually lie in areas other than the striatum. Thus, the augmentation of DA levels in the SN produced by L-DOPA administration was found to correlate more precisely with its behavioral actions when compared to L-DOPA-induced changes in striatal DA levels (Robertson and Robertson, 1989). Indeed, studies have shown that repeated systemic administration of L-DOPA will induce several alterations in the electrophysiological activity of SN neurons, including the reversal of lesion-induced supersensitivity of GABAergic zona reticulata neurons to DA agonists (Weick et al., 1990) and a decrease in the sensitivity of DA neurons to DA agonist-mediated inhibition in intact rats (Jackson et al., 1982). On this basis, this study examines whether a portion of the therapeutic benefits of L-DOPA therapy in an animal model of Parkinson's disease may be mediated by changes oc- curring within the SN. This was done by examining the effects of acute and repeated L-DOPA administration on the electrophysiological activity of DA neurons in rats with 6-OHDA-induced lesions of the striatal DA innervation.

A portion of these results have been published in abstract form (Harden and Grace, 1993).

\section{Materials and Methods}

All experiments were performed on male Sprague-Dawley rats (ZivicMiller Laboratories, Allison Park, PA). Animal care and surgical procedures were performed in accordance with the guidelines outlined in the NIH Guide for the Care and Use of Laboratory Animals and were approved by the Institutional Animal Care and Use Committee of the University of Pittsburgh.

6-OHDA-induced DA lesions. Rats weighing 250-300 gm were anesthetized with Equithesin $(0.3 \mathrm{ml} / 0.1 \mathrm{~kg})$ and pretreated with desmethylimipramine (DMI; Sigma; $25 \mathrm{mg} / \mathrm{kg}$ i.p.) to protect noradrenergic terminals from damage (Breese and Traylor, 1971). The rats were mounted in a Kopf rat stereotaxic apparatus fitted with nonpuncture ear bars. Local anesthetic (1\% lidocaine; Elkins-Sinn, Cherry Hill, NJ) was infused into wounds and pressure points. The skull was exposed, and two small burr holes were made bilaterally in the bone overlying the lateral ventricles ( $1.5 \mathrm{~mm}$ lateral to midline, $1 \mathrm{~mm}$ posterior to bregma). Twenty-five minutes following pretreatment with DMI, stainless steel cannulae $(0.6 \mathrm{~mm}$ o.d.) were lowered simultaneously into the lateral ventricles $(3.2 \mathrm{~mm}$ ventral to the dura). Ten milliliters of a solution containing $100 \mu \mathrm{g}$ of 6-OHDA dissolved in $3 \%$ ascorbic acid was slowly infused into each lateral ventricle over a $15 \mathrm{~min}$ period using a syringe pump (Model 341, Sage). Ten minutes following the completion of the infusion the cannulae were withdrawn, the burr holes sealed with bone wax, and the midline incision closed with sutures. Body temperature was maintained postoperatively using a thermostatically controlled heating pad until the animals recovered from anesthesia and exhibited spontaneous mobility. Body weight was monitored daily, and animals were provided with palatable mash (Heinz baby food mixed with chocolate powder in warm water) ad libitum to compensate for lesion-induced aphagia, which is known to occur following extensive DA lesions (Zigmond and Stricker, 1973). A 5\% dextrose/saline solution was administered i.p. to rats if any evidence of dehydration was observed.

$\mathrm{L}-D O P A$ administration. On the eighth postoperative day, at which time 6-OHDA-induced DA cell death should be maximal (Onn et al., 1986; Nakazato and Akiyama, 1988), one group of DA-depleted animals began receiving twice daily injections of L-DOPA (L-DOPA methylester; Sigma) $(50 \mathrm{mg} / \mathrm{kg}$ ) plus benserazide (a peripheral decarboxylase inhibitor; Hoffmann-LaRoche; $50 \mathrm{mg} / \mathrm{kg}$ ). A second group of DA-depleted animals were given twice daily injections of isotonic saline $(0.9 \%)$ to parallel the L-DOPA treatments. After a minimum of 4 weeks of L-DOPA or saline treatment, half of the animals from each group received an injection of isotonic saline and half received L-DOPA + benserazide $(50 \mathrm{mg} / \mathrm{kg})$ approximately $20 \mathrm{~min}$ before receiving anesthesia in preparation for electrophysiological recordings. This treatment protocol resulted in four 6-OHDA lesion groups: (1) repeated saline followed by acute saline (saline-saline), (2) repeated saline followed by acute L-DOPA (saline-L-DOPA), (3) repeated L-DOPA followed by acute saline (L-DOPA-saline), and (4) repeated L-DOPA followed by acute L-DOPA (L-DOPA-L-DOPA) (see Table 1). A total of 48 lesioned rats (12 per group) that met the critera for DA cell loss were recorded in this study. In addition, recordings were made from 12 intact, untreated rats to allow for calculation of the relative proportion of spontaneously active DA cells, as well as for comparison of basal firing rate and firing pattern.

Electrophysiological recording. Extracellular single-unit recordings were performed in chloral hydrate-anesthetized rats $(400 \mathrm{mg} / \mathrm{kg}$ i.p.) using procedures similar to those described previously (Hollerman and Grace, 1990). Briefly, the deeply anesthetized rats were mounted in a stereotaxic apparatus and the lateral tail vein catheterized for supplemental administration of anesthetic. Extracellular single-unit recordings of DA neuron spike activity were obtained using glass microelectrodes pulled from $2.0 \mathrm{~mm}$ o.d. Omegadot glass tubing (WPI) using a vertical microelectrode puller (Narishige). Electrodes were broken back under microscopic control to achieve a tip diameter of approximately $1 \mu \mathrm{m}$; the electrodes were then filled with $2 \mathrm{M} \mathrm{NaCl}$ containing $2 \%$ Pontamine sky blue, yielding impedances of 5-10 $\mathrm{M} \Omega$ measured in vitro at 135 Hz. Signals were amplified by a high-impedance headstage amplifier 
Table 1. Effects of repeated saline or L-DOPA treatment on the proportion of spontaneously active DA neurons

\begin{tabular}{llll} 
Treatment & $\begin{array}{l}\text { DA neurons } \\
\text { recorded in nine } \\
\text { tracks (mean } \\
\pm \text { SEM) }\end{array}$ & $\begin{array}{l}\text { \% of fluorescent } \\
\text { neurons remaining }\end{array}$ & $\begin{array}{l}\text { Proportion of DA } \\
\text { neurons firing }\end{array}$ \\
\hline Intact & $11.75 \pm 0.98$ & $100 \%$ & $3.1 \pm 0.30$ \\
Saline-Saline & $1.75 \pm 0.45$ & $31.8 \pm 3.6 \%$ & $4.7 \pm 1.70$ \\
Saline-L-DOPA & $2.33 \pm 0.53$ & $32.7 \pm 3.0 \%$ & $5.56 \pm 1.46$ \\
L-DOPA-Saline & $6.83 \pm 0.41 \dagger$ & $32.5 \pm 1.9 \%$ & $17.2 \pm 1.10 \dagger$ \\
L-DOPA-L-DOPA & $5.67 \pm 0.91 \ddagger$ & $34.2 \pm 2.3 \%$ & $13.40 \pm 3.52 \ddagger$ \\
\hline
\end{tabular}

"The proportion of spontaneously active DA neurons was obtained by dividing the number of DA cells exhibiting spontaneous spike activity recorded in nine tracks by the number of DA neurons histologically counted.

i $\mathrm{L}$-DOPA-saline vs saline-saline, $p<0.01$.

†-DOPA-L-DOPA vs saline-saline, $p<0.01$.

connected to a preamplifier (Fintronics, Orange, CT) and were monitored on a Kikusui Storage Oscilloscope (COS50520-ST) and a Grass Instruments AM-8 audio monitor. Action potentials were isolated using a time and amplitude window discriminator (Fintronics) and spike frequency was integrated over $10 \mathrm{sec}$ bins.

Midbrain DA neurons have been shown to alter their activity along three dimensions in response to a challenge: (1) firing rate, (2) firing pattern, and (3) the relative proportion of cells firing spontaneously (Bunney and Grace, 1978; Grace and Bunney, 1986). In order to sample these parameters across comparable populations of DA cells in different animals, the electrophysiological activity of spontaneously firing DA neurons was sampled in the central region of the SN zona compacta by making nine vertical passes of the electrode through the DA cell body region beginning at $2.9 \mathrm{~mm}$ anterior to the lambdoid suture and $2.2 \mathrm{~mm}$ lateral to midline, with each electrode track separated ${ }^{\prime}, 0.2 \mathrm{~mm}$ in the anterior-posterior and medial-lateral dimensions. The entire region of the SN sampled extended from 2.1-3.1 mm anterior to the lambdoid suture and $2.0-2.4 \mathrm{~mm}$ lateral to midline, and contained the DA neurons that project primarily to the dorsal striatum (Guyenet and Aghajanian, 1978). Recordings were begun approximately $45 \mathrm{~min}$ after acute administration of saline or L-DOPA. The nine-track protocol was completed approximately $2 \mathrm{hr}$ after the acute drug injection. Spontaneously active DA neurons were identified on the basis of previously established criteria, which include their location within the stereotaxically defined region of the $\mathrm{SN}$, the presence of characteristic long-duration (1.8-4.5 msec) biphasic action potentials exhibiting a prominent negative phase and typically showing a notch on the rising phase of the spike, and a relatively slow firing rate $(1-9 \mathrm{~Hz})$ with spikes occurring in a slow irregular single-spiking or burst-firing pattern (Bunney et al., 1973; Grace and Bunney, 1980, 1983). The parameters of activity sampled consisted of (1) the number of spontaneously firing DA neurons encountered per electrode track, (2) the basal firing rate sampled over a 5 min period, and (3) the proportion of spikes fired by a neuron that occurred within bursts. At least 5 min of spontaneous spike activity for each neuron was digitized and recorded onto VHS videocassettes using a Neurncorder (Neurodata, New York); subsequent analyses were then performed off line. Quantitative analysis of the burst-firing pattern was performed using a Cambridge Electronic Design 1401 Laboratory Computer Interface and the Spike2 software package (Cambridge Electronics Design, Cambridge, UK) run on a MS/DOS microcomputer system. As described previously (Grace and Bunney, 1984b), the initiation of a burst of spikes was defined as the occurrence of two spikes wilh an interspike interval of less than $80 \mathrm{msec}$ and the end of a burst identified by the occurrence of consecutive spikes with an interspike interval of $160 \mathrm{msec}$ or greater.

Estimating extent of DA depletion. Because administration of L-DOPA was assumed to alter basal levels of tissue DA in treated rats (Hefti et al., 1981), the standard procedure for calculating lesion size based on percent loss of striatal tissue DA levels (Keller et al., 1976) could not be accurately employed. Instead, a correlation analysis relating the percent of tissue DA loss to the number of DA cells remaining in the SN as determined histologically was performed, which was similar to that used in a previous study (Hollerman and Grace, 1990). As in the previous report, the proportion of histologically identified DA cells remaining in the SN was determined at the completion of the nine- track protocol by processing the $\mathrm{SN}$ for catecholamine histofluorescence using the sucrose-phosphate-glyoxylic acid (SPG) procedure (de la Torre and Surgeon, 1976). Briefly, the deeply anesthetized rats were removed from the stereotaxic, decapitated, and their brains rapidly removed. A $4 \mathrm{~mm}$ block containing the SN was frozen at $-15^{\circ} \mathrm{C}$ and 25 $\mu \mathrm{m}$ thick sections were cut using a cryostat (Reichert Histostat). Every fifth section cut from the midbrain was collected on a glass slide, dipped in SPG solution, dried under a stream of air, and subsequently placed in a $95^{\circ} \mathrm{C}$ oven for $150 \mathrm{sec}$ to yield the fluorescent reaction product. Fluorescent neurons were counted manually in every treated section using a Leitz Orthoplan II epifluorescence microscope equipped with a Leitz D filter cube. The criterion for counting a cell in a section was the presence of a distinguishable soma. The proportion of DA cells lesioned at three different midbrain levels was determined using the average cell counts obtained from two adjacent sections corresponding to the anterior ( $3.4 \mathrm{~mm}$ anterior to lambdoid suture), central $(3.0 \mathrm{~mm}$ anterior to lambda), and posterior ( $2.6 \mathrm{~mm}$ anterior to lambda) regions of the SN. The extent of DA neuron loss was then calculated by dividing the number of DA neurons observed at each level in 6-OHDAtreated animals by the average number of DA neurons counted in sections taken from two animals that had not been subjected to the 6-OHDA lesion.

Statistics. All statistical analyses were performed using SYSTAT 5.0 computer software. Statistical analysis included ANOVA followed by post hoc Fisher's least significant difference. For data not satisfying the criteria for parametric tests, Kruskal-Wallis followed by post hoc Mann-Whitney $U$ analyses were performed. Firing rate distributions were evaluated using the nonparametric Kolmogorov-Smirnov test. All results are reported as mean \pm SEM.

\section{Results}

Histological assessment of DA lesion size

The method that is typically employed to assess the extent of DA depletion produced by 6-OHDA treatment is HPLC analysis of striatal tissue DA content (Keller et al., 1976). However, because repeated L-DOPA treatment has been shown to result in the accumulation of DA within striatal tissues (Hefti et al., 1981), this method was not considered to be a reliable measure of the extent of the DA lesion in this case, particularly when comparing striata of rats from saline versus L-DOPA treatment groups. Thus, in the present study, lesion size was calculated histologically on the basis of DA cell loss rather than by biochemical measures. A 50\% DA cell loss was established as the criterion for inclusion of electrophysiological data for statistical analysis, since previous studies have shown that this degree of cell loss corresponds to: (1) a loss of approximately $80 \%$ of striatal DA (Hollerman and Grace, 1990); and (2) the proportion of DA cells that must be lesioned before systemic administration of DA agonists will elicit lateralized behavioral activation in rats with unilateral DA depletions (Cole et al., 1993; Hudson et al., 


\begin{abstract}
Table 2. Effects of acute and repeated $\ell$-DOPA administration on basal firing rate and firing pattern of spontaneously active DA neurons
\end{abstract}

\begin{tabular}{llc} 
Treatment & $\begin{array}{l}\text { Mean firing } \\
\text { rate } \pm\end{array}$ & $\begin{array}{l}\text { Mean percentage } \\
\text { of spikes fired in } \\
\text { bursts } \pm \text { SEM }\end{array}$ \\
\hline Intact & $4.0 \pm 0.15$ & $18.4 \pm 1.9 \%$ \\
Saline-saline & $4.4 \pm 0.40$ & $15.5 \pm 4.0 \%$ \\
Saline-L-DOPA & $2.9 \pm 0.20^{* \dagger}$ & $5.3 \pm 1.1 \% \neq$ \\
L-DOPA-Saline & $4.6 \pm 0.20$ & $23.6 \pm 2.5 \%$ \\
L-DOPA-L-DOPA & $3.8 \pm 0.20 \S$ & $8.6 \pm 1.2 \% \S$ \\
\hline
\end{tabular}

* Saline-saline vs saline-L-DOPA, $p<0.01$.

$\uparrow$ Saline-L-DOPA vs L-DOPA-L-DOPA, $p<0.01$.

\$ Saline-saline vs saline-L-DOPA, $p<0.02$.

$\S$ L-DOPA-saline vs L-DOPA-L-DOPA, $p<0.01$

1993). Overall, 79 rats were used in this study. Out of a total 61 animals that received 6-OHDA injections, a total of 48 animals (12 per treatment group) met the criterion of an overall loss of $50 \%$ of DA cells, with cell loss for all of the rats tested ranging from $51-87 \%$. These results are analogous to those obtained previously in which the i.c.v. infusion of 6-OHDA resulted in a greater loss of DA cell somata within the posterior regions of the $\mathrm{SN}$ as compared to more anterior regions (Hollerman and Grace, 1990). No significant difference in the total number of DA neurons remaining was found when comparing results from rats that had received repeated administration of saline with those treated repeatedly with L-DOPA, with the mean percentage of DA cells lost in each group ranging from 66-68\% $(F=0.14 ; p>0.93$; Table 1$)$. Thus, L-DOPA treatment begun 1 week following 6-OHDA infusion did not appear to either enhance or prevent DA cell death induced by 6-OHDA treatment.

\section{Effects of L-DOPA on DA cell activity states}

Basal firing rate. DA neurons in control intact rats exhibited an average basal firing rate of $4.0 \pm 0.15 \mathrm{~Hz}$ (range $=0.8-9.7 \mathrm{~Hz}$; $n=141$ cells). The average firing rate of DA neurons recorded in lesioned rats following repeated saline ( $n=24$ cells) administration was not significantly different from intact rats (salinesaline $=4.4 \pm 0.4 \mathrm{~Hz}$ vs intact $=4.0 \pm 0.1 \mathrm{~Hz} p>0.30)$. Similarly, in rats receiving repeated L-DOPA treatment $(n=74$ cells), the mean basal firing rate of DA neurons was not significantly different from the saline-treated animals (saline-saline $=$ $4.4 \pm 0.4 \mathrm{~Hz}$ vs L-DOPA-saline $=4.6 \pm 0.2 \mathrm{~Hz} ; p>0.60$; Table 2). In contrast, in animals that had been treated repeatedly with saline, the average firing rate of the $\mathrm{DA}$ cells recorded following an acute dose of L-DOPA ( $n=36$ cells) was significantly slower than that of rats receiving acute saline (salinesaline $=4.4 \pm 0.4 \mathrm{~Hz}$ vs saline-L-DOPA $=2.9 \pm 0.2 \mathrm{~Hz} ; p$ $<0.01$; Fig. 1). Similarly, in animals receiving repeated L-DOPA treatment the DA neurons recorded after a subsequent acute dose of L-DOPA ( $n=70$ cells) had a significantly slower mean firing rate than those receiving acute saline (L-DOPAsaline $=4.6 \pm 0.2 \mathrm{~Hz}$ vs L-DOPA-L-DOPA $=3.8 \pm 0.2 \mathrm{~Hz}$; $p<0.01)$. Furthermore, the firing rate of neurons recorded in the saline-L-DOPA group were significantly $(p<0.01)$ slower than those of the L-DOPA-L-DOPA group. In order to compare the magnitude of inhibition observed following acute L-DOPA administration, the difference in mean firing rates between sa-

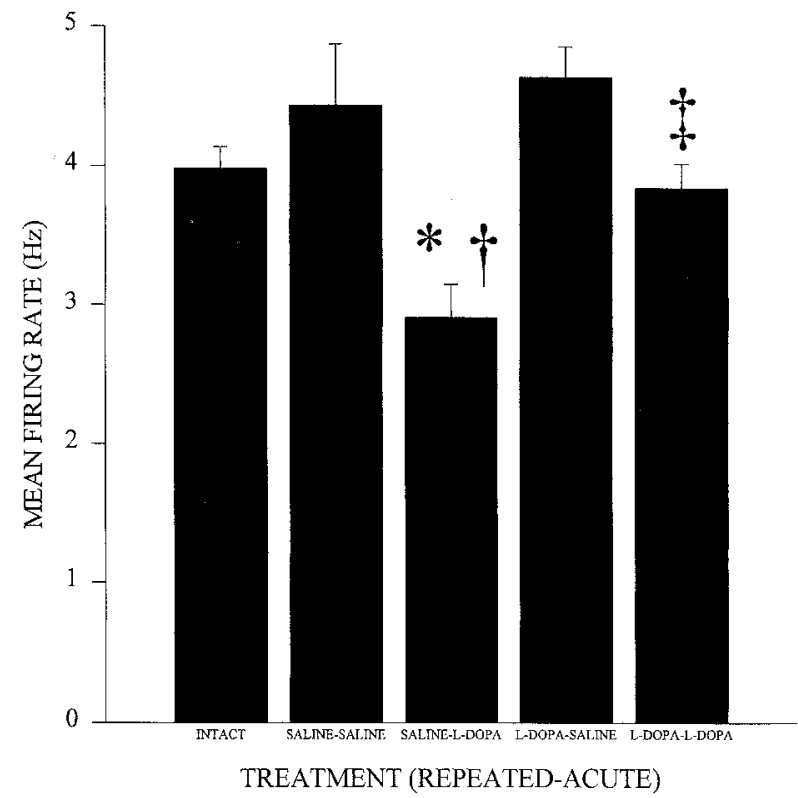

Figure 1. Efrects of DA lesions on the firing rate of DA neurons. In DA-depleted animals the mean firing rate of all DA neurons recorded following repeated saline treatment (saline-saline) was not significantly different from that recorded in control (intact) animals. Similarly, following repeated L-DOPA treatment (L-DOPA-saline) the mean firing rate was not significantly different from that observed in saline pretreated animals. In contrast, acute administration of L-DOPA following saline pretreatment (saline-L-DOPA) or L-DOPA pretreatment (L-DOPA$\mathrm{L}-D O P A$ ) resulted in significantly slower mean firing rates as compared to saline challenge. After repeated L-DOPA administration, however, the decrease in mean firing rate observed following acute L-DOPA was significantly less than the decrease observed in the saline pretreated animals. Symbols used in the figure refer to the following comparisons: $\left.{ }^{*}\right)$ saline-saline vs saline-L-DOPA, $p<0.01$; $(\dagger)$ L-DOPA-saline versus L-DOPA-L-DOPA, $p<0.01$; (†) L-DOPA-L-DOPA versus salineL-DOPA, $p<0.01$.

line-saline and saline-L-DOPA was compared with the difference between L-DOPA-saline and L-DOPA-L-DOPA. This analysis revealed that an acute dose of L-DOPA produced significantly less inhibition in these L-DOPA-pretreated rats when compared to the L-DOPA-induced decrease in average firing rate of salinepretreated animals ( $p<0.02$; Fig. 1). In addition to exhibiting a slower mean firing rate, the DA neurons recorded in salinetreated rats after acute administration of L-DOPA also showed a significantly different distribution of firing rates across cells; i.e., the distribution of firing rates was skewed toward lower firing rates as compared to the saline-saline treatment group (Kolmogorov-Smirnov $p<0.02$ ). In contrast, acute L-DOPA administration did not result in a significant alteration in the distribution of firing rates in the rats that had received repeated L-DOPA treatment (Kolmogorov-Smirnov $p>0.30$; Fig. 2).

Firing pattern. DA neurons in control intact rats fired primarily in an irregular, single-spiking pattern with $18.4 \pm 1.9 \%$ (range $=0.0-94.2 \%$ ) of all spikes fired occurring in bursts. In lesioned rats treated repeatedly with saline, the amount of burst firing was not significantly different from intact (saline-saline $=15.5 \pm 4.0 \%$ vs intact $=18.4 \pm 1.9 ; \mathrm{p}$; Fig. 3 ), and this parameter was not altered in the I.-DOPA-treated rats (I.-DOPAsaline $=23.6 \pm 2.5 \%$ vs intact $=18.4 \pm 1.9 \% ; p>0.10)$. However, when rats received an acute injection of L-DOPA immediately prior to recording from either the saline- or L-DOPApretreated rats, the DA cells fired significantly less of their 
SALINE-SALINE

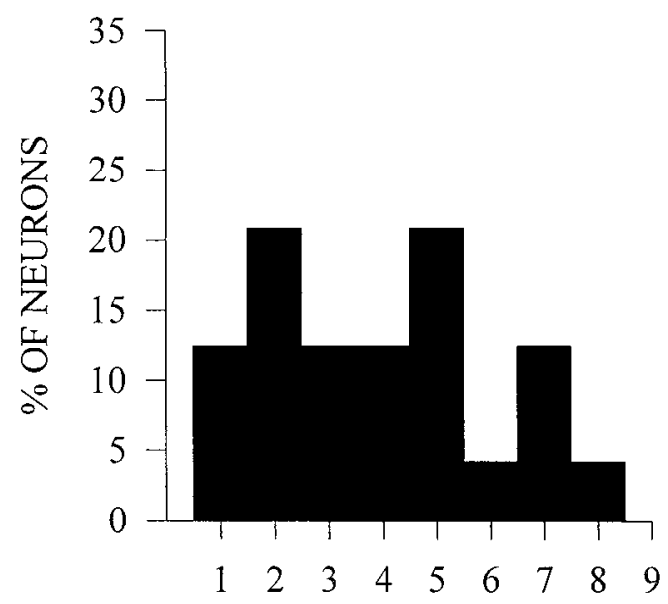

FIRING RATE $(\mathrm{Hz})$
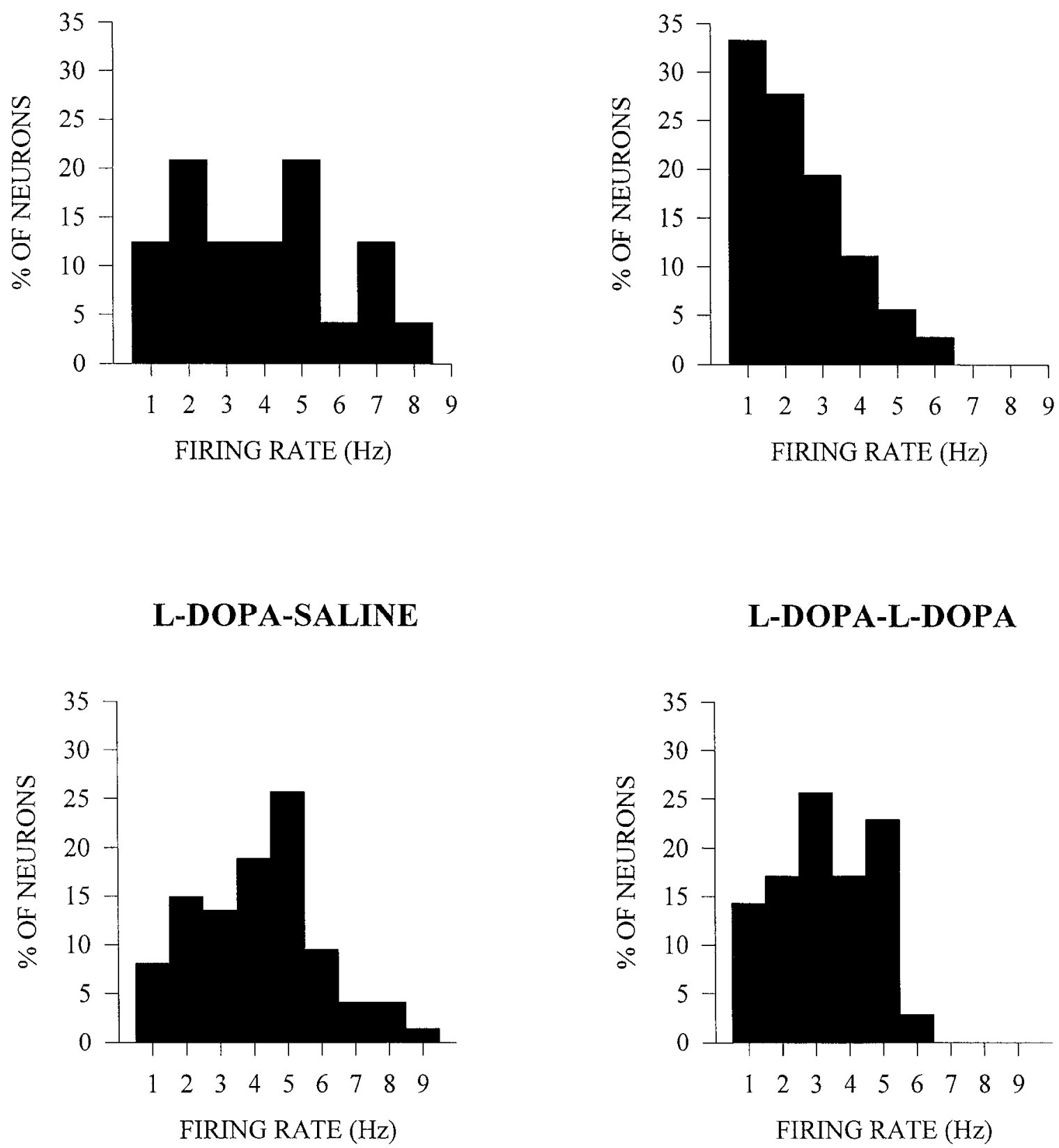

FIRING RATE $(\mathrm{Hz})$

\section{L-DOPA-L-DOPA}

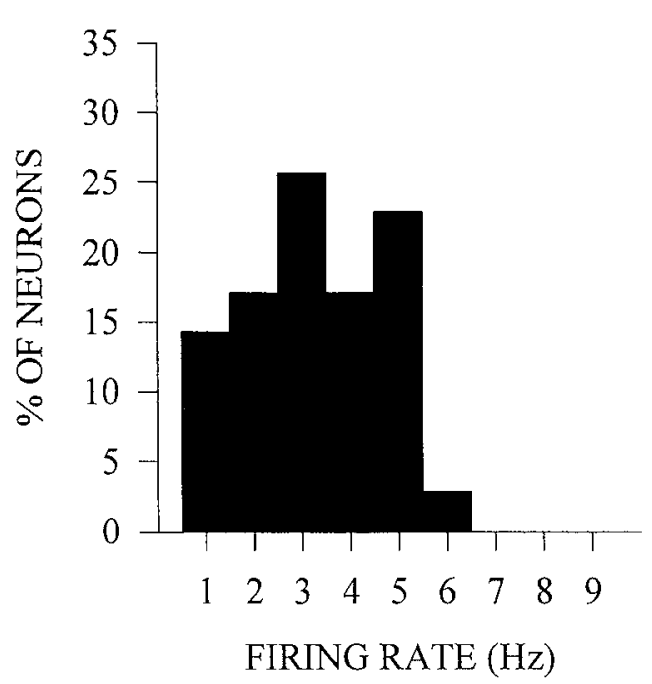

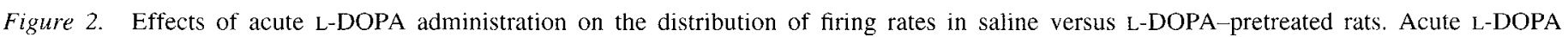

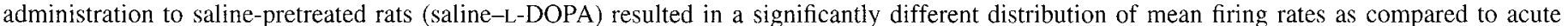

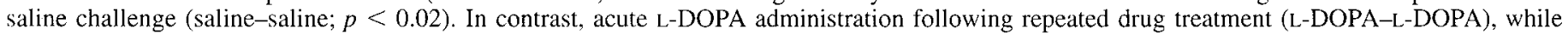

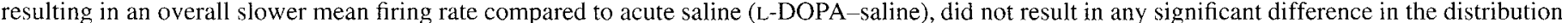
of firing frequencies $(p>0.30)$.

spikes in bursts (saline-saline $=15.5 \pm 4.0 \%$ vs saline-L-DOPA $=5.3 \pm 1.1 \% ; p<0.02 ; \mathrm{L}-\mathrm{DOPA}-$ saline $=23.6 \pm 2.5 \%$ vs L-DOPA-L-DOPA $=8.6 \pm 1.2 \% ; p<0.01)$. Thus, in contrast to the effect produced on firing rate, the acute administration of L-DOPA caused a similar degree of inhibition of burst firing when comparing rats that had received L-DOPA pretreatment with those that had been treated with saline (difference between saline-saline and saline L-DOPA vs L-DOPA-saline L-DOPAL-DOPA; $p>0.08$ ).

Spontaneous activity. In control nonlesioned rats, passing the electrode through a defined region of the $\mathrm{SN}$ in a predetermined nine-track pattern yielded an average of $11.8 \pm 1.0$ cells $/$ nine tracks. In contrast, in DA-depleted animals receiving repeated saline treatment, the average number of DA cells recorded in nine tracks was $1.8 \pm 0.4$ cells (saline-saline vs intact, $p<$ 0.01 ). The DA-depleted animals that had received repeated L-DOPA treatment had a significantly greater number of cells firing spontaneously; i.e., $6.8 \pm 0.4$ active DA neurons in nine tracks (L-DOPA--saline vs saline-saline, $p<0.01$; Table 1). Saline-pretreated rats that had received an injection of L-DOPA on 


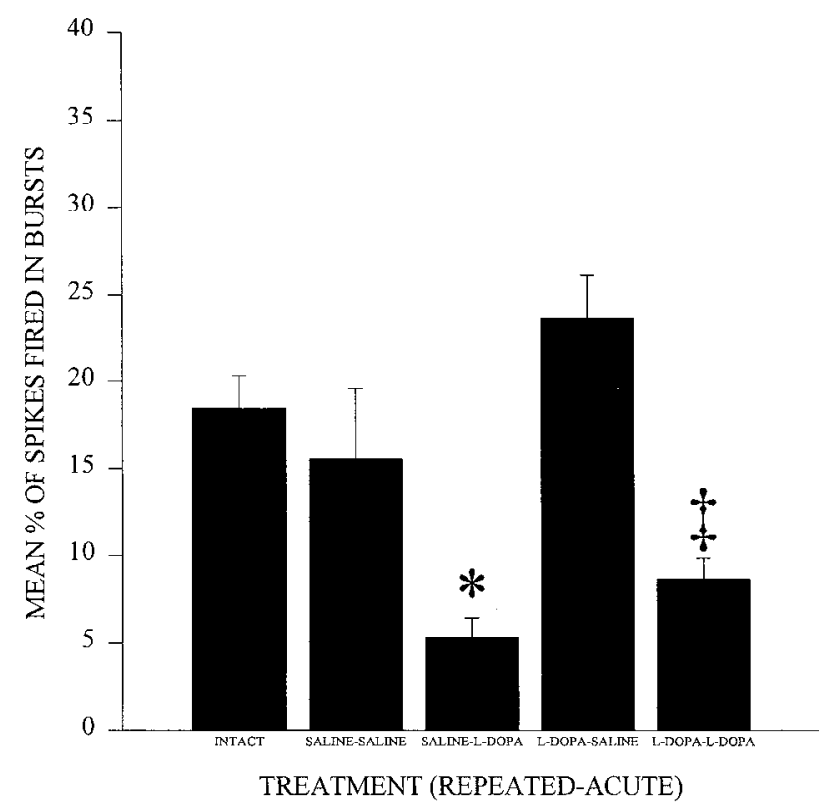

Figure 3. DA neurons recorded in DA-depleted rats that had received repeated saline treatment (saline-saline) or repeated L-DOPA treatment (L-DOPA-saline) did not exhibit significant differences in the mean percentage of spikes fired in bursts as compared to control (intact) animals. Acute L-DOPA administration following either repeated saline (salineL-DOPA) or repeated L-DOPA (L-DOPA-L-DOPA) administration did result in a significant reduction in the mean percentage of spikes fired in bursts. In contrast to its effect on firing rate, following repeated L-DOPA administration the reduction in burst firing observed following acute drug challenge (L-DOPA-L-DOPA) was not significantly different from that observed in saline-pretreated rats (saline-L-DOPA). Symbols used in the figure refer to the following comparisons: $(*)$ saline-saline versus saline-L-DOPA, $p<0.02$ and ( $\$$ ) $\mathrm{L}-\mathrm{DOPA}-$ saline versus $\mathrm{L}-$ -DOPA-L-DOPA, $p<0.01$

the day of recording did not exhibit significant differences in the number of spontaneously active DA neurons encountered (saline-saline $=1.8 \pm 0.4$ neurons/nine tracks vs saline-L-DOPA $=2.3 \pm 0.5$ cells/nine tracks; $p>0.30$ ). Similarly, in rats that had received repeated L-DOPA treatment, the number of spontaneously firing DA cells was the same, regardless of whether the rats were challenged with acute L-DOPA or acute saline on the day of recording $(\mathrm{L}-\mathrm{DOPA}-\mathrm{saline}=6.8 \pm 0.4$ cells $/$ nine tracks vs L-DOPA-L-DOPA $=5.7 \pm 0.9$ cells $/$ nine tracks; $p>$ $0.20)$.

Relative proportion of spontaneously active DA neurons. Although significant differences in the number of spontaneously active cells were observed following repeated drug administration, it is possible that this may be due to differences in lesion size or to some trophic factor of L-DOPA treatment, even though overall the percentage of cells lost was not significantly different between groups $(F=0.14 ; p>0.93$; Table 1$)$. Therefore, the values calculated for the number of active cells were corrected for each animal by dividing it by the percentage of histologically identified DA cells remaining, yielding a number that should be related to the proportion of the remaining neurons showing spontaneous spike firing. Application of this correction showed that there was a highly significant increase in the proportion of spontaneously active DA neurons following repeated L-DOPA treatment relative to the nonlesioned, nontreated intact animals ( $\mathrm{L}$ DOPA-saline $=17.2 \pm 1.1 \mathrm{vs}$ intact $3.1 \pm 0.3 ; p<0.01$; L-DOPA-L-DOPA $=13.40 \pm 3.52$ vs intact $=3.1 \pm 0.3 ; p<$
$0.02)$ and in comparison to DA-depleted, saline-treated animals $(\mathrm{L}-\mathrm{DOPA}-\mathrm{saline}=17.2 \pm 1.1 \mathrm{vs}$ saline-saline $=4.7 \pm 1.7 ; p$ $<0.01$; Table 1). In agreement with our previous study (Hollerman and Grace, 1990), moderate levels of DA depletion alone did not result in a significant change in the relative of proportion of spontaneously active DA neurons (intact $=3.1 \pm 0.3$ vs saline-saline $=4.7 \pm 1.7 ; p>0.30$ ).

\section{Discussion}

Parkinson's disease is characterized by a loss of nigrostriatal DA neurons (Hornykiewicz, 1966). This movement disorder can be at least temporarily relieved by a drug chosen for its ability to replenish central DA levels; i.e., the DA precursor L-DOPA (Lloyd et al., 1975). However, the therapeutic efficacy of this drug is limited in terms of its extended use and is known to produce severe side effects (see Klawans et al., 1990, for review). Furthermore, initial studies by others have provided evidence suggesting that the ability of L-DOPA to restore behavioral function may involve mechanisms more complex than simply the restoration of DA levels in the striatum (Spencer and Wooten, 1984).

One quandary regarding the mode of action of L-DOPA relates to the dissociation between the time course of the increase in extracellular DA levels and the behavioral recovery occurring during the course of repeated L-DOPA administration. For example, several studies have shown that in unilaterally DA-depleted rats, behavioral activation induced by L-DOPA administration outlasts the elevation in DA observed in the lesioned striatum (Spencer and Wooten, 1984; Robertson and Robertson, 1989). Furthermore, the increase in extracellular DA in the DAdepleted striatum following a single administration of L-DOPA has been shown to remain constant during a course of repeated treatments, even though the hehavioral effects were observed to increase with successive doses of the drug (Wachtel and Abercrombie, 1993). Therefore, the effects of L-DOPA-induced changes on DA levels within the striatum appear to be insufficient to completely account for the therapeutic actions of this drug.

In this study, we used administration of the DA-specific neurotoxin 6-OHDA to provide an animal model of Parkinson's disease to test the short- and long-term consequences of L-DOPA treatment. Our results provide evidence supporting a role for L-DOPA-induced restoration of DA cell spike activity in the behavioral recovery that develops during treatment.

\section{Measures of DA cell activity}

In order to provide an estimate of the electrophysiological activity of a population of midbrain DA neurons, previous studies have measured DA cell activity along three dimensions: (1) basal firing rate, (2) firing pattern, and (3) number of spontaneously active DA neurons (Bunney and Grace, 1978; Hollerman and Grace, 1990). Although DA neurons exhibit changes in firing rates upon drug administration, alterations in this parameter are believed to be comparatively less effective at altering DA release due to the relatively slow rates of spike discharge and narrow range of firing frequencies exhibited by these cells (i.e., 1-10 $\mathrm{Hz}$; Grace and Bunney, 1984a). On the other hand, studies have provided evidence that DA cells may increase DA output by altering their firing pattern. Thus, DA cells fire in two distinct patterns: irregular single-spike firing and burst firing (Grace and Bunney, 1984a,b). Gonon (1988) has demonstrated that DA cells can increase the amount of DA released per spike by two- to 
threefold when they change from a single-spiking to a burstfiring pattern. Furthermore, since studies have shown that up to $50 \%$ of the DA cells in the SN exist in a hyperpolarized, nonspiking state (Bunney and Grace, 1978; Grace and Bunney, 1986), DA release may also be controlled by altering the proportion of DA cells tiring spontaneously, as measured by passing an electrode through the $\mathrm{SN}$ in a predetermined pattern and counting the number of active DA cells encountered.

\section{$D A$ cell activity states-differential effects of acute versus repeated $\mathrm{L}-D O P A$ administration}

The DA cells recorded in DA-depleted rats treated with saline (saline-saline) exhibited levels of activity similar to those of intact rats in terms of their basal firing rates, firing patterns, and proportion of spontaneously active DA neurons, which is consistent with the results of our previous study (Hollerman and Grace, 1990). Furthermore, DA cells in rats treated repeatedly with L-DOPA (L-DOPA-saline) also exhibited basal firing rates and firing patterns that were not significantly different from those found in intact rats. However, after repeated L-DOPA treatment, a significantly higher proportion of DA neurons were found to be firing spontaneously (L-DUPA-L-DOPA and L-DOPAsaline). Since only two time points (acute and 4 weeks) during the course of L-DOPA administration were assessed with respect to DA cell activity, we cannot state precisely at what point the repeated L-DOPA caused an activation of DA neuron firing. Given that the delayed behavioral actions of L-DOPA occur within 7-8 d of treatment, its effects on DA neuron firing are also likely to occur well within the 4 week assessment period.

In contrast to rats that had received repeated L-DOPA treatment, acute L-DOPA administration (saline-L-DOPA) failed to cause a significant change in the proportion of DA neurons exhibiting spontaneous activity, but did result in significant alterations in the basal firing rate and firing pattern of DA neurons that were spontaneously discharging. Thus, in the salinepretreated rats, DA cells recorded following acute L-DOPA administration had significantly slower mean firing rates, as well as a decrease in the mean percentages of spikes fired in bursts. After repeated L-DOPA administration, a subsequent dose of the drug (L-DOPA-L-DOPA) still resulted in significantly lower firing rates and burst firing; however, the magnitude of the decrease in firing rate was significantly less in L-DOPA-pretreated rats than that produced in saline-pretreated rats. Thus, the effectiveness of acute L-DOPA administration in producing slower basal firing rates appears to be significantly reduced following repeated drug treatment. In contrast, the magnitude of the reduction of burst firing in response to acute L-DOPA was similar in both pretreatment conditions (i.e., saline-LDOPA and L-DOPA-L-DOPA).

\section{Interpretation of $\mathrm{L}-D O P A$ treatment effects}

The results presented in this study demonstrate that the effects produced by acute L-DOPA administration on DA cell activity are substantially different from those produced by repeated L-DOPA treatment. Repeated administration of L-DOPA could conceivably exert this activation of DA cell firing at two sites of action: on DA cell autoreceptors or on striatonigral feedback pathways innervating the DA neuron. However, the majority of evidence appears to favor a site of action at the DA cell autoreceptor: (1) it is consistent with data showing that the time course of L-DOPA-induced increases in DA levels in the SN correlate more closely to behavior than do the changes occurring in the striatum (Robertson and Robertson, 1989; Orosz and Bennett, 1992); (2) the preponderance of evidence shows that changes in autoreceptor stimulation is correlated with changes in the number of DA neurons firing; in contrast, this parameter is little affected by striatonigral lesions (Chiodo and Bunney, 1983); (3) a continued presence of high levels of dopamine in the striatum would be expected to result in a diminishing effect of dopaminc on striatal cells (and, hence, striatonigral feedback) rather than an increasing action; and (4) conceptually, it is presumed that the clinically relevant change occurring during L-DOPA treatment is ultimately a change in striatal activity secondary to changes in DA cell firing; therefore, a change in striatal activity should be the result rather than the cause of activation of dopaminergic neurons.

Based on the analysis of firing rate and burst firing, acute L-DOPA administration appears to have similar effects on firing rate and firing pattern to that of other DA autoreceptor agonists (Bunney et al., 1973; Skirboll et al., 1979). It is likely that inhibition of DA cell firing by acute L-DOPA administration occurs via stimulation of the somatodendritic autoreceptor (Mercuri et al., 1992). Indeed, drawing on the finding that DA is synthesized and released from the dendrites of DA neurons (Geffen et al., 1976; Korf et al., 1976), recent studies have suggested that some of the therapcutic actions associated with L-DOP $\Lambda$ treatment may be mediated within the SN. Support for this hypothesis comes from the results of several studies demonstrating that L-DOPA administration produces a robust increase in extracellular DA levels in the DA-depleted SN, and that the time course of the behavioral effects of L-DOPA is better correlated with this L-DOPA-induced increase in SN DA levels than with the changes in DA levels that occur in the striatum (Robertson and Robertson, 1989; Orosz and Bennett, 1992).

Previously, our laboratory had demonstrated that, following partial DA depletions, the remaining DA neurons exhibited an enhanced sensitivity to DA agonists, and, furthermore, that this effect was due to an increase in the sensitivity of DA cell somatodendritic autoreceptors to systemically administered DA agonists (Pucak and Grace, 1991). Such an increase in autoreceptor sensitivity could therefore account for the enhanced response of DA neurons in lesioned, saline-pretreated rats to the acute administration of L-DOPA. In contrast, after repeated L-DOPA administration, the acute inhibitory actions of L-DOPA on DA cells appears to be significantly reduced with respect firing rate. This suggests that the enhanced sensitivity of the DA autoreceptors observed following DA depletion is reversed during the course of repeated L-DOPA administration. This model is consistent with the findings of Jackson et al. (1982), in which a significant reduction in the sensitivity of DA neurons to the inhibitory effects of DA agonists was observed following repeated L-DOPA administration to intact rats. In contrast, acute L-DOPA results in a similar reduction in burst firing in both saline-pretreated and L-DOPA-treated animals. This finding is likely to be related to studies suggesting that the firing pattern of DA neurons is regulated by several different factors. Thus, burst firing in $\mathrm{SN}$ can be decreased both by autoreceptor stimulation (Bunney et al., 1973; Grace and Bunney 1984b) or by lesions of the glutamatergic afferents arising from the subthalamic nucleus (Smith and Grace, 1992). The participation of afferent processes in the modulation of burst firing is further supported by studies showing that DA cells do not fire in a bursting pattern in the in vitro midbrain slice preparation (Grace and Onn, 1989). Thus, unlike the strong dependence of L-DOPA-induced inhibition of 
DA cell firing on autoreceptor sensitivity, it is likely that several factors may influence the actions of L-DOPA on the burst firing pattern.

Pharmacological data suggest that the somatodendritic autoreceptors may control at least two dimensions of DA cell activity. First, acute DA autoreceptor stimulation exerts an inhibitory effect on the basal firing rate and the amount of burst firing of these ncurons (Skirboll et al., 1979). Sccond, the proportion of DA neurons that are firing spontaneously appears to depend on the ability of endogenous DA to stimulate somatodendritic autoreceptors. This proposal is supported by several findings: (1) acute blockade of DA autoreceptors by haloperidol increases the number of spontaneously active DA neurons recorded per electrode track (Bunney and Grace, 1978); (2) repeated administration of amphetamine increases the number of spontaneously firing DA neurons only during the period when there is a concomitant decrease in autoreceptor sensitivity (White and Wang, 1984a,b); and (3) an observed increase in autoreceptor sensitivity following DA depletion has been proposed to underlie the absence of feedback activation that accompanies DA depleting lesions, and which is circumvented by subsequent blockade of these autoreceptors (Hollerman and Grace, 1989; Pucak and Grace, 1991).

\section{Summary and conclusions}

In this study, we provide correlative evidence that some of the delayed therapeutic benefits of L-DOPA may occur via an activation of normally nonfiring DA cells. However, one question that remains is the reason for the lack of correspondence between DA cell firing and striatal DA levels. This may be accounted for by consideration of the functional differences between spike-dependent DA release and extrasynaptic DA levels, as elaborated in a recent model (Grace, 1991). The release of DA from terminals within the striatum has been proposed to occur via two distinct mechanisms: a spike-dependent phasic DA release, which is large in amplitude but is rapidly removed from the synapse by reuptake into the DA terminal before it can escape from the synaptic cleft; and (2) a steady-state tonic DA release, which is maintained in the extrasynaptic space in the striatum (Grace, 1991). Studies have shown that the spike-dependent, phasic DA release appears to mediate the behavioral actions of the DA system (Hollerman and Grace, 1989; Grace, 1991), and often occurs in the absence of measurable changes in extrasynaptic DA levels (see Grace, 1991). On the other hand, the tonic DA levels in the extrasynaptic space appear to be the DA pool that is measured by microdialysis, and which exhibits steady-state increases following acute L-DOPA administration (Wachtel and Abercrombie, 1993). Given the dissociation between the rapid effects of L-DOPA on rigidity versus its delayed actions on the motor control (Hardie, 1990; Gancher, 1992; Juncos, 1992), one possibility is that the rapid restoration of tonic DA levels by acute L-DOPA administration may underlie the immediate antirigidity actions of the drug, whereas the delayed increase in DA cell spike activity are needed for recovery of advanced motor control. This proposed dependence of motor function on $D \wedge$ cell spike induced $D \Lambda$ release rather than on tonic DA receptor stimulation is further supported by studies of MPTP-treated monkeys in which the therapeutic effects of either D1 or D2 agonists were completely blocked by pretreatment with the DA synthesis inhibitor $\alpha$-methyl-p-tyrosine (GomezMancilla and Bédard, 1991). Thus, although DA agonists may supply DA receptors with additional stimulation during their ad- ministration, the fact that recovery can be disrupted by DA synthesis inhibition supports a critical role for spike-dependent DA release in their therapeutic actions. We propose that L-DOPA acts in a similar manner.

On the other hand, L-DOPA appears to achieve this action by circumventing an important mode of DA cell regulation: i.e., local feedback via the inhibitory somatodendritic autoreceptor. Indecd, circumvention of DA autorceptor fecdback by haloperidol administration to rats that had recovered from DA depletion is thought to be the primary factor leading to the appearance of profound akinesia secondary to depolarization block of DA cell firing (Hollerman and Grace, 1989; Hollerman et al., 1992). In a similar manner, by desensitizing the autoreceptor, L-DOPA administration may yield therapeutic actions at the expense of stability within this system. Under these conditions, factors that place demand upon this system are likely to cause the DA cells to rapidly enter depolarization block (Bunney and Grace, 1978; Hollerman and Grace, 1989). Such a rapidly occurring transition into and out of depolarization block could conceivably lead to such side effects as on/off effects or end-ofdose anomalies that are known to occur during L-DOPA treatment of Parkinson's disease (see Klawans et al,, 1990). Indeed, the ability of low doses of apomorphine to reverse on/off effects in parkinsonian patients (Pocwc ct al., 1988a; Stibe ct al., 1988) at doses similar to those required to reverse DA cell depolarization block in rats (Hollerman and Grace, 1989) is consistent with this model. On the other hand, therapeutic approaches that target activation of DA cell firing or augmentation of the postsynaptic response to synaptic DA release may be effective at treating Parkinson's disease without the concomitant side effects known to occur with traditional L-DOPA therapy.

\section{References}

Acheson AL, Zigmond MJ, Stricker EM (1980) Compensatory increase in tyrosine hydroxylase activity in rat brain after intraventricular injection of 6-hydroxydopamine. Science 207:537-540.

Agid Y, Javoy F, Glowinski J (1973) Hyperactivity of remaining dopaminergic neurons after partial destruction of the nigrostriatal dopaminergic system in the rat. Nature New Biol 245:150-151.

Bernheimer H, Birkmayer W, Hornykiewicz O (1973) Brain dopamine and the syndromes of Parkinson and Huntington: clinical, morphological and neurochemical correlates. J Neurol Sci 20:415-455.

Breese GR, Traylor TD (1971) Depletion of brain noradrenaline and dopamine by 6-hydroxydopamine. Br J Phamacol 42:88-89.

Bunney BS, Grace AA (1978) Acute and chronic haloperidol treatment: comparison of effects on nigral dopamine cell activity. Life Sci $23: 1 \% 15-1728$.

Bunney BS, Walters JR, Roth RH, Aghajanian GK (1973) Dopaminergic neurons: effect of antipsychotic drugs and amphetamine on single cell activity. J Pharmacol Exp Ther 185:560-571.

Chiodo LA, Bunney BS (1983) Typical and atypical neuroleptics: differential effects of chronic administration on the activity on A9 and A10 midbrain dopaminergic neurons. J Neurosci 3:1607-1619.

Cole DG, Growdon JH, DiFiglia M (1993) Levodopa induction of Fos immunoreactivity in rat brain following partial and complete lesions of the substantia nigra. Exp Neurol 120:223-232.

Creese I, Synder SH (1979) Nigrostriatal lesions enhance striatal ${ }^{3} \mathrm{H}-$ apomorphine and ${ }^{3} \mathrm{H}$-spiroperidol binding. Eur J Plarmacol 56:277281.

Creese I, Burt DR, Snyder SH (1977) Dopamine receptor binding enhancement accompanies lesion-induced behavioral supersensitivity. Science 197:596-598.

de la Torre JC, Surgeon JW (1976) A methodological approach to rapid and sensitive monoamine histofluorescence using a modified glyoxylic acid technique: the SPG method. Histochemistry 49:81-93.

Gancher ST (1992) Pharmacology of Parkinson's Disease. In: Parkinson's disease neurobehavioral aspects (Huber ST, Cummings JL, eds), pp 273-287. New York: Oxford UP. 
Geffen LB, Tessel TM, Cuello AC, Iverson LL (1976) Release of dopamine from dendrites in rat substantia nigra. Nature 260:258-260.

Gomez-Mancilla, Bædard PJ (1991) Effect of D1 and D2 agonists and antagonists on dyskinesia produce by L-DOPA in 1-methyl-4-phenyl1,2,3,6-tetrahydropyridine-treated monkeys. J Pharmacol Exp Ther 259:409-413.

Gonon FG (1988) Nonlinear relationship between impulse flow and dopamine released by rat midbrain dopaminergic neurons as studied by in vivo electrochemistry. Neuroscience 24:19-28.

Grace AA (1991) Phasic versus tonic dopamine release and the modulation of dopamine system responsivity: a hypothesis for the etiology of schizophrenia. Neuroscience 41:1-24.

Grace AA, Bunney BS (1980) Nigral dopamine neurons: intracellular recording and identification with L-DOPA injection and histofluorescence. Science 210:654-656.

Grace AA, Bunney BS (1983) Intracellular and extracelluar electrophysiology of nigral dopaminergic neurons-1. Identification and characterization. Neuroscience 10:301-315.

Grace AA, Bunney BS (1984a) The control of firing pattern in nigral dopamine neurons: single spike firing. J Neurosci 4:2866-2876.

Grace AA, Bunney BS (1984b) The control of firing pattern in nigral dopamine neurons: burst firing. J Neurosci 4:2877-2890.

Grace $\Lambda \Lambda$, Bunney BS (1985) Opposing effects of striatonigral feedback pathways on midbrain dopamine cell activity. Brain Res 333: 271-284.

Grace AA, Bunney BS (1986) Induction of depolarization block in midbrain dopamine neurons by repeated administration of haloperidol: analysis using in vivo intracellular recording. J Pharmacol Exp Ther 238:1092-1100

Grace AA, Onn S-P (1989) Morphology and electrophysiological properties of immunocytochemically identified tat dopamine neurons recorded in vitro. J Neurosci 9:3463-3481.

Guyenet PG, Aghajanian GK (1978) Antidromic identification of dopaminergic and other output neurons of the rat substantia nigra. Brain Res 150:69-84.

Harden DG, Grace AA (1993) Repeated L-DOPA administration increases the number of spontaneously active A9 dopamine neurons in the partial dopamine depleted rat. Soc Neurosci Abstr 19:1583.

Hardie RJ (1990) Chapter 20: levodopa-related motor fluctuations. In Parkinson's Disease (Stern GM, ed), pp 559-596. Baltimore: The Johns Hopkins UP.

Hefti F, Melamed E, Wurtman RJ (1980) Partial lesions of the dopaminergic nigrostriatal system in rat brain: biochemical characterization. Brain Res 195:69-84.

Ilefti F, Melamed E, Wurtman RJ (1981) The site of dopamine formation in rat striatum after L-DOPA administration. $\mathbf{J}$ Pharmacol Exp Ther 217:189-197.

Hollerman JK, Grace AA (1989) Acute haloperidol administration induces depolarization block of nigral dopamine neurons in rats after partial dopamine lesions. Neurosci Lett 96:82-88.

Hollerman JR, Grace AA (1990) The effects of dopamine-depleting brain lesions on the electrophysiological activity of rat substantia nigra dopamine neurons. Brain Res 533:203-212.

Hollerman JR, Abercrombie ED, Grace AA (1992) Electrophysiological, biochemical, and behavioral studies of acute haloperidol-induced depolarization block of nigral dopamine neurons. Neuroscience 47: 589-601.

Hornykiewicz O (1966) Dopamine (3-hydroxytyramine) and brain function. Pharmacol Rev 18:925-964

Hudson JL, Horne CGV, Stromberg I, Brock S, Clayton J, Masserano J, Hoffer BJ, Gerhardt GA (1993) Correlation of apomorphine- and amphetamine-induced turning with nigrostriatal dopamine content in unilateral 6-hydroxydopamine lesioned rats. Brain Res 626:167-174.

Jackson DM, Walters JR, Miller LP (1982) Chronic L-DOPA-pretreatment of rats: an electrophysiological and biochemical study in the basal ganglia. Brain Res 250:271-282.

Juncos JL (1992) Levodopa: pharmacokinetics and pharnacodynamics. Neurol Clin 10:487-450.

Keller R, Oke A, Mefford I, Adams RN (1976) Liquid chromotagraphic analysis of catecholamines: routine assay for regional brain mapping. Life Sci 19:995-1004.

Klawans H, Hitri A, Barr A (1990) Levodopa-induced dyskinesias. In: Parkinson's disease (Stera G, ed), pp 537-557. Baltimore: Johns Hopkins UP.
Korf J, Zieleman M, Westernick BHC (1976) Dopamine release in the substantia nigra? Nature 260:257-258.

Lloyd KG, Davidson L, Hornykiewicz O (1975) The neurochemistry of Parkinson's disease: effect of L DOPA therapy. J Pharmacol Exp Ther 195:453-464.

Marshall JF, Teitelbaum P (1973) A comparison of the eating in response to hypothermic and glucoprivic challenges after nigral 6-hydroxydopamine and lateral hypothalamic electrolytic lesions in rats. Brain Res 55:229-233.

Mercuri NB, Calabresi P, Bernardi G (1992) The electrophysiological actions of dopamine and dopaminergic drugs on neurons of the substantia nigra pars compacta and ventral tegmental area. Life Sci 51 : 711-718.

Nakazato T, Akiyama A (1988) In vivo voltammetric study of 6-hydroxydopamine-induced neuronal degradation. J Neurochem 51 $1007-1013$.

Nisenbaum ES, Sticker EM, Zigmond MJ, Berger TW (1986) Longterm effects of dopamine-depleting brain lesions on spontaneous activity of Type II striatal neurons: relation to behavioral recovery. Brain Res 398:221-230.

Nisenbaum ES, Stricker EM, Zigmond MJ, Berger IW (1988) Spontaneous activity of Type II but not Type I striatal neurons is correlated with recovery of behavioral function after dopamine-depleting lesions. Brain Res 473:389-393.

Onn S-P, Berger TW, Stricker EM, Zigmond MJ (1986) Effects of intraventricular 6-hydroxydopamine on the dopaminergic innervation of striatum: histochemical and neurochemical analysis. Brain Res $376: 8-19$.

Orosz D, Bennett JP (1992) Simultaneous microdialysis in striatum and substantia nigra suggests that the nigra is a major site of action of L-dihydroxyphenylalaninie in the "hemiparkinsonian" rat. Exp Neurol 1 is:388-393.

Orr WB, Gardiner TW, Sticker EM, Zigmond MJ, Berger TW (1986) Short-term effects of dopamine-depleting brain lesions on spontaneous activity of striatal neurons: relation to local dopamine concentration and behavior. Brain Res 376:20-28.

Poewe W, Kleedorfer B, Gersentbrandt F, Oerterl W (1988a) Subcutaneous apomorphine in Parkinson's disease. Lancet 1:943.

Pucak ML, Grace AA (1991) Partial dopamine depletions result in and enhanced sensitivity of residual dopamine neurons to apomorphine. Synapse 9:144-155.

Robertson GS, Robertson HA (1989) Evidence that L-DOPA-induced rotational behavior is dependent on both striatal and nigral mechanisms. J Neurosci 9:3326-3331.

Skirboll LR, Grace AA, BS Bunney (1979) Dopamine auto- and postsynaptic receptors: electrophysiological evidence for differential sensitivity to dopamine agonists. Science 206: 80-82.

Smith ID, Grace AA (1992) Role of subthalamic nucleus in the regulation of nigral dopamine neuron activity. Synapse 12:287-303.

Spencer SE, Wooten GF (1984) Pharmacologic effects of L-DOPA are not closely linked temporally to striatal dopamine concentration. Neurology 34:1609-1611.

Stachowiak MK, Keller RWJ, Sticker EM, Zigmond MJ (1987) Increased dopanime efflux from striatal slices during development and after nigrostriatal bundle damage. J Neurosci 7:1648-1654.

Stibe CM, Kempster PA, Lees AJ, Stern GM (1988) Subcutaneous apomorphine in Parkinsonian on-off oscillations. Lancet 1:403-406.

Wachtel SR, Abercrombie ED (1993) Long-term L-DOPA administration to rats with 6-OHDA lesions: behavior and striatal neurochemistry. Soc Neurosci Abstr 19:1370.

Weick BG, Engber TM, Susel Z, Chase TN, Walters JR (1990) Responses of substantia nigra pars reticulata neurons to GABA and SKF 38393 in 6-hydroxydopamine-lesioned rats are differentially affected by continuous an intermittent levodopa administration. Brain Res 523:16-22.

White FJ, Wang RY (1984a) Al0 dopamine neurons: role of autoreceptors in determining firing rate and sensitivity to dopamine agonists. Life Sci 34:1161-1170.

White FJ, Wang RY (1984b) Electrophysiological evidence for A10 dopamine autoreceptor subsensitivity following chronic $d$-amphetamine treatment. Brain Res 309:283-292.

Zhang WQ, Tilson HA, Nanry KP, Hudson PM, Hong JS, Stachowiak MK (1988) Increased dopamine release from striata of rats after unilateral nigrostriatal bundle damage. Brain Res 461:335-342.

Zigmond MJ, Stricker E M (1973) Recovery of feeding and drinking 
by rats after intraventricular 6-hydroxydopamine or lateral hypothalamic lesions. Science 182:717-720.

Zigmond MJ, Stricker EM (1984) Parkinson's disease: studies with an animal model. Life Sci 35:5-18.

Zigmond MJ, Acheson AL, Stachowiak MK, Stricker EM (1984) Neurochemical compensation after nigrostriatal bundle injury in an animal model of preclinical parkinsonism. Arch Neurol 41:856861 .

Zigmond MJ, Berger TW, Grace AA, Stricker EM (1989) Compensatory responses to nigrostriatal bundle injury: studies with 6-hydroxydopamine in an animal model of parkinsonism. Mol Chem Neuropathol 10:185-200. 